\title{
ESTIMATIVA DO CONSUMO HÍDRICO DA GOIABEIRA, UTILIZANDO ESTAÇÕES AGROMETEOROLÓGICAS AUTOMÁTICA E CONVENCIONAL ${ }^{1}$
}

\author{
ANTÔNIO HERIBERTO DE CASTRO TEIXEIRA² ${ }^{2}$, LUÍS HENRIQUE BASSOI², VALDECIRA CARNEIRO DA SILVA \\ REIS $^{3}$, THIERES GEORGE FREIRE DA SILVA ${ }^{4}$, MARCELO DE NOVAES LIMA FERREIRA ${ }^{5}$, JOSELANNE LUIZA \\ TRAJANO MAIA ${ }^{6}$
}

RESUMO - A evapotranspiração (ETc) e o coeficiente de cultura $(\mathrm{Kc})$ da goiabeira (Psidium guajava L.) cv. Paluma, aos dois anos e três meses após o plantio e irrigada por microaspersão, foram determinados durante um ciclo de produção, em Petrolina-PE, Brasil. O método da razão de Bowen foi utilizado para a estimativa da evapotranspiração da cultura (ETc), enquanto o de Penmam-Monteith foi empregado para a estimativa da evapotranspiração de referência (ETo), com a utilização de duas estações agrometeorológicas, uma automática e outra convencional. A ETc acumulada entre a poda (junho de 2001) e a colheita (dezembro de 2001) foi de $906 \mathrm{~mm}$ em 200 dias, correspondendo a um valor médio de 4,53 $\pm 0,68 \mathrm{~mm}$. dia ${ }^{-1}$. O Kc apresentou valores entre 0,75 a 0,93 e de 0,61 a 0,84 , quando foram usadas as estações convencional e automática, respectivamente.

Termos para indexação: Psidium guajava L., balanço de energia, evapotranspiração, coeficiente de cultura.

\section{EVALUATION OF WATER CONSUMPTION OF GUAVA TREES BY AUTOMATIC AND CONVENTIONAL AGROMETEOROLOGICAL STATIONS}

\begin{abstract}
The crop evapotranspiration (ETc) and the crop coefficient (Kc) of guava cv. Paluma irrigated with a microsprinkler were estimated for two years and three months after planting and over one growing season at Petrolina, Pernambuco State, Brazil. The ETc was estimated by Bowen ratio, and the reference evapotranspiration (ETo) was estimated using data collected by conventional and automatic agrometeorological stations. The accumulated ETc from pruning (June 2001) to harvest (December 2001) was $906 \mathrm{~mm}$ in 200 days, which corresponds to an average of $4.53 \pm 0.68 \mathrm{~mm}$ day${ }^{1}$. Kc varied from 0.75 to 0.93 when the conventional station was used, and from 0.61 to 0.84 when using an automatic station.
\end{abstract}

Index terms: Psidium guajava L., energy balance, evapotranspiration, crop coefficient.

\section{INTRODUÇÃO}

No Vale do São Francisco, em Petrolina-PE e Juazeiro-BA, a goiabeira é uma das culturas frutíferas mais exploradas, com uma área de plantas em formação e produção superior a 3.700 ha (CODEVASF, 1999). Pode-se ter o início de produção dois anos após o plantio, com duas safras por ano, sendo a cultivar Paluma a mais plantada (Gonzaga Neto et al., 1991).

A importância de estudos sobre evapotranspiração das espécies frutíferas no Vale do São Francisco deve-se à necessidade, por parte dos fruticultores, da adoção de critérios definidos no local para o manejo da irrigação, com o uso eficiente de água e energia.

O conceito de evapotranspiração de referência (ETo) refere-se à transferência do vapor d'água de uma área gramada para a atmosfera. A grama deve estar em crescimento ativo, mantida a uma altura uniforme de 0,08 a $0,12 \mathrm{~m}$, sombreando completamente o solo e sem deficiência de água. A evapotranspiração de uma cultura (ETc) acontece quando as plantas se encontram em áreas extensas, sem a incidência de pragas, doenças e deficiência nutricional, com disponibilidade de água que não seja limitante ao desenvolvimento, e de modo que o potencial de produção seja atingido, para a condição climática em que se encontram. A razão entre a ETc e ETo origina o coeficiente de cultura $(\mathrm{Kc})$, que depende do estádio de desenvolvimento das plantas, do sistema de irrigação, da configuração de plantio e das condições meteorológicas reinantes (Allen et al., 1998).

No Vale do São Francisco, a estimativa de ETo vem sendo obtida através de dados de estações agrometeorológicas convencionais, porém as estações automatizadas estão sendo difundidas junto aos produtores. Segundo Sentelhas et al. (1997), o tipo de estação meteorológica utilizado para a medição de elementos climáticos deve ser considerado, pois, para um mesmo elemento, existem diferenças nos resultados obtidos por estações convencionais e automáticas, principalmente em escala diária.

Dessa forma, há a necessidade de comparação entre os dados obtidos por esses diferentes tipos de estações. Assim, o presente trabalho teve como objetivo a obtenção e comparação dos valores de coeficiente de cultura $(\mathrm{Kc})$ no decorrer das fases fenológicas da goiabeira irrigada em Petrolina- PE, com a utilização de estações agrometeorológicas convencionais e automáticas.

\section{MATERIALEMÉTODOS}

O experimento foi conduzido no Campo Experimental da Embrapa Semi-Árido, em Petrolina-PE (latitude 09 $09^{\circ} \mathrm{S}$, longitude 40 $22^{\prime}$ W, altitude $365,5 \mathrm{~m}$ ). O clima da região é do tipo BSwh', segundo a classificação de Köeppen, que corresponde a uma região árida. A cultura da goiabeira (Psidium guajava L.), cv. Paluma, foi plantada em março de 1999, em um Latossolo Vermelho-Amarelo, textura média (Pereira \& Souza, 1967). O espaçamento da cultura foi de 5 X 6m, e o sistema de irrigação utilizado foi o de microaspersão. O manejo de irrigação baseouse no monitoramento do potencial matricial $\left(\Psi_{\mathrm{m}}\right)$ do solo por tensiômetros instalados nas profundidades de 20;40;60;80 e $100 \mathrm{~cm}$. O manejo de irrigação baseou-se no monitoramento do potencial matricial $\left(\Psi_{\mathrm{m}}\right)$ do solo por tensiômetros instalados nas profundidades de 20;40;60;80 e $100 \mathrm{~cm}$. O momento de irrigação foi determinado quando o potencial matricial atingiu o valor próximo a - $0,03 \mathrm{MPa}$, na profundidade efetiva das raízes $(60 \mathrm{~cm})$ (Bassoi et al., 2002). Os valores de umidade do solo $(\theta)$ correspondente aos valores de $\Psi$ foram obtidos pelas curvas de retenção de água em cada profundidade de instalação dos tensiômetros. As curvas foram determinadas em laboratório com amostras deformadas de solo e descritas pelo modelo de Van Genutchen (1980). A lâmina de irrigação a ser aplicada foi determinada para elevar $\theta$ próximo à capacidade de campo $\left(\Psi_{\mathrm{m}}=-0,01 \mathrm{MPa}\right)$, e o tempo de irrigação foi estimado pelas expressões:

\footnotetext{
${ }^{1}$ (Trabalho 183/2002). Recebido: 21/08/2002. Aceito para publicação: 11/09/2003. Financiado pelo CNPq - Programa Nordeste de Pós-Graduação e Pesquisa. ${ }^{2}$ Pesquisador, Embrapa Semi-Árido, C. P. 23, CEP 56302-970, Petrolina-PE, Telefone: (87) 38621711 heribert@cpatsa.embrapa.br, lhbassoi@cpatsa.embrapa.br. ${ }^{3}$ Eng. Agrônomo, Fazenda Fruitfort, Projeto de Irrigação Senador Nilo Coelho N-2, CEP 56300-000, Petrolina-PE.

${ }^{4}$ Graduando em Agronomia, UNEB-DCTS, Av. Edgard Chastinet, s/n, CEP 48900-000, Juazeiro-BA.

${ }^{5}$ Pós-graduando em Irrigação e Drenagem, ESALQ-USP, Depto Eng. Rural, C. P. 9, CEP 13418-900, Piracicaba-SP.

${ }^{6}$ Bolsista do CNPq, Embrapa Semi-Árido.
} 
$\mathrm{L}_{\mathrm{L}}=\left(\theta_{\mathrm{cc}}-\theta_{\text {atual }}\right) \mathrm{Z}$

$\mathrm{Ti}=\frac{\text { LLE1 E2 P }}{\text { Ein Q }}$

em que LLé a lâmina líquida de irrigação $(\mathrm{mm}) ; \theta_{\mathrm{cc}}$ a umidade do solo à capacidade de campo $\left(\mathrm{m}^{3} \mathrm{~m}^{-3}\right) ; \theta_{\text {atual }}$ a umidade atual do solo $\left(\mathrm{m}^{3} \mathrm{~m}^{-3}\right) ; \mathrm{Z}$ a profundidade da camada de solo considerada $(60 \mathrm{~mm})$; Ti o tempo de irrigação (h); E1 e E2 os espaçamentos entre plantas e entre linhas de plantas, respectivamente $(6 \mathrm{~m} \times 5 \mathrm{~m})$; P a fração de solo molhada pelos microaspersores $(0,42) ; \mathrm{E}_{\mathrm{i}}$ a eficiência do sistema de irrigação $(0,9) ; \mathrm{n} o$ número de microaspersores por planta (1), e Q é a vazão do microaspersor $\left(\mathrm{L} . \mathrm{h}^{-1}\right)$, medida em teste de campo.

Para a determinação da ETc, a partir de dados do saldo de radiação, do fluxo de calor no solo, dos gradientes de temperatura do ar $(\Delta \mathrm{T})$ e temperatura do bulbo úmido ( $\Delta \mathrm{TU})$, e da equação do balanço de energia (equação 3 ), foram obtidos os fluxos de calor latente de evaporação (LE) (equação 4) e de calor sensível H (equação 5), empregando-se a razão de Bowen ( $\beta$ ) (equação 6), segundo Webb (1965):

$\mathrm{Rn}+\mathrm{LE}+\mathrm{G}+\mathrm{H}=0$
$\mathrm{LE}=-\frac{\mathrm{Rn}+\mathrm{G}}{1+\beta}$
$\mathrm{H}=-(\mathrm{Rn}+\mathrm{LE}+\mathrm{G})$
$\beta=\left(\frac{\left.\ddot{\mathrm{A}}+\gamma \frac{\ddot{\mathrm{ATU}}}{\gamma}-1\right)^{-1}}{\ddot{\mathrm{A} T}}\right.$

sendo Ä a tangente à curva de saturação do vapor d'água no $\operatorname{ar}\left(\mathrm{mb}{ }^{\circ} \mathrm{C}-\right.$ ${ }^{1}$ ) em função da temperatura média dos termômetros de bulbo úmido, entre os dois níveis, onde os psicrômetros foram instalados, e $\gamma$ é o coeficiente psicrométrico $\left(0,66 \mathrm{mb}^{\circ} \mathrm{C}\right)$.

Para o cálculo de ETo pelo método de Penman-Monteith, foram utilizados dados obtidos nas estações agrometeorológicas convencional e automática (Campbell), junto à área experimental, e a seguinte equação (Allen et al., 1998):

ETo $=\frac{0,408 \ddot{A}(\mathrm{Rn}-\mathrm{G})+\gamma \frac{900}{\mathrm{~T}+273} \mathrm{U}_{2}\left(\mathrm{e}_{\mathrm{s}}-\mathrm{e}_{\mathrm{a}}\right)}{\ddot{\mathrm{A}}+\gamma\left(1+0,34 \mathrm{U}_{2}\right)}$

em que ETo é a evapotranspiração de referência (mm.dia $\left.{ }^{-1}\right)$; Rn é o saldo de radiação à superfície $\left(\mathrm{MJ} \cdot \mathrm{m}^{2} \cdot \mathrm{dia}^{-1}\right)$; G é o fluxo de calor sensível no solo $\left(\mathrm{MJ} \mathrm{m}^{-2} \mathrm{dia}^{-1}\right)$; T a temperatura média do ar $\left({ }^{\circ} \mathrm{C}\right) ; \mathrm{U}_{2}$ a velocidade do vento a $2 \mathrm{~m}$ de altura $\left(\mathrm{m} . \mathrm{s}^{-1}\right) ;\left(\mathrm{e}_{\mathrm{s}}-\mathrm{e}_{\mathrm{a}}\right)$ o déficit de pressão do vapor d'água $(\mathrm{kPa}) ; \Delta$ a declividade da curva de pressão de vapor de saturação $(\mathrm{kPa}$ ${ }^{\circ} \mathrm{C}^{-1}$ ), e 900 um fator de conversão. A declividade da curva da pressão de saturação do vapor foi obtida em função da temperatura média do ar (média aritmética entre as temperaturas máximas e mínimas diárias).

Para a estimativa do saldo de radiação sobre a superfície de referência, foi utilizada a seguinte fórmula:

$\mathrm{Rn}=\mathrm{Rns}-\mathrm{Rnl}$

sendo Rns e Rnl os saldos de radiação de ondas curtas e ondas longas, respectivamente.

Por sua vez, estes foram obtidos através das expressões:

Rns $=(1-\alpha) \operatorname{Rs}$

onde $\alpha$ é o coeficiente de reflexão que é 0,23 para a superfície de grama de referência e Rs a radiação solar global incidente.

$\mathrm{Rnl}=o ́\left(\underline{\mathrm{T}}^{4} \frac{\max , \mathrm{K}+\mathrm{T}^{4}}{2} \underline{\min , \mathrm{K}}\right)\left(0,34-0,14 \mathrm{ve}_{\mathrm{a}}\right)\left(1,35 \frac{\mathrm{Rs}}{\text { Rso }}-0,35\right)(10)$

em que ó é a constante de Stefan Boltzmann $\left(4,903 \cdot 10^{-9} \mathrm{M} \mathrm{J} \mathrm{K}^{-4} \mathrm{~m}^{-2} \mathrm{dia}^{-1}\right)$;
Tmax,K e Tmin,K são as temperaturas máximas e mínimas no período de 24 horas $\left(\mathrm{K}={ }^{\circ} \mathrm{C}+273,16\right)$; e é a pressão atual do vapor d'água $(\mathrm{kPa})$; $\mathrm{Rs}$ é a radiação solar global incidente ( $\left.\mathrm{M} \mathrm{J} \mathrm{m}^{-2} \mathrm{dia}^{-1}\right)$, e Rso é a radiação solar global incidente em dias totalmente sem nuvens ( $\left.\mathrm{M} \mathrm{J} \mathrm{m}^{-2} \mathrm{dia}^{-1}\right)$.

Na estação automática, as temperaturas máximas e mínimas foram obtidas programando-se o sistema de aquisição de dados para armazenar os valores máximos e mínimos em 24 horas, enquanto, na estação convencional, essas temperaturas foram provenientes de leituras diárias dos termômetros de máxima e de mínima.

A pressão atual do vapor d'água na estação automática foi calculada da seguinte forma:

$\mathrm{e}_{\mathrm{a}}=\frac{\mathrm{e}_{\mathrm{s}}(\operatorname{Tmin}) \frac{\mathrm{URmax}}{100}+\mathrm{e}_{\mathrm{s}}(\operatorname{Tmax}) \frac{\mathrm{URmin}}{100}}{2}$

onde $\mathrm{e}_{\mathrm{s}}(\operatorname{Tmin})$ e $\mathrm{e}_{\mathrm{s}}$ (Tmax) são as pressões de saturação do vapor d'água às temperaturas mínimas e máximas, respectivamente; URmáx e URmín são os valores máximos e mínimos para a umidade relativa do ar no período de 24 horas, obtidos também por meio da programação do sistema de aquisição de dados.

Na estação convencional, a pressão atual do vapor foi obtida através da expressão:

$\left.\mathrm{e}_{\mathrm{a}}=\frac{\mathrm{URmed}}{100} \frac{(\operatorname{Tmax}+\mathrm{Tmin}}{2}\right)$

onde URmed é a média compensada da umidade relativa do ar, obtida por meio de psicrômetro com aspiração, para os três horários diários de observações agrometeorológicas sinópticas.

Para a obtenção da radiação solar global incidente (Rs) na estação convencional, realizou-se a planimetria diária de diagramas de actinógrafo bimetálico, enquanto, na estação automática, se obteve esse parâmetro através do piranômetro acoplado ao sistema de aquisição de dados.

A radiação solar global para dia de céu claro foi calculada por :

Rso $=(a+b)$ Ra

sendo $\mathrm{Ra}$ a radiação solar incidente no topo da atmosfera e $(\mathrm{a}+\mathrm{b}) \mathrm{a}$ fração dessa radiação que chega na superfície terrestre em dias claros. Os coeficientes a e b utilizados para cada mês foram os obtidos por Teixeira (1999).

Com os dados climáticos provenientes tanto da estação agrometeorológica automática como da convencional, foram obtidos pela relação ETc/ETo os valores de Kc para a cultura da goiabeira, no período compreendido desde a poda, em 7 de junho de 2001, até o término da colheita, em 24 de dezembro de 2001. Na colheita, o peso dos frutos foi determinado para a estimativa da produção $\left(\mathrm{kg} \mathrm{ha}^{-1}\right)$. A eficiência do uso de água (EUA) foi estimada pela relação entre a produção (kg ha${ }^{1}$ ) e a quantidade de água evapotranspirada (mm).

\section{RESULTADOSEDISCUSSÃO}

Entre a poda de frutificação e o término da colheita, a evapotranspiração acumulada da cultura foi de $906 \mathrm{~mm}$ (valor médio de 4,53 $\pm 0,68 \mathrm{~mm} \mathrm{dia}^{-1}$, e o diâmetro de copa variou de 3 a 4,7 m. A produção de frutos foi de $24.097 \mathrm{~kg} \mathrm{ha}^{-1}$, o que resulta em uma EUA de 26,6 $\mathrm{kg} \mathrm{ha}^{-1}$ $\mathrm{mm}^{-1}$. As fases fenológicas da goiabeira estão apresentadas na Tabela 1 .

A Figura 1 apresenta as médias decendiais da evapotranspiração da cultura, a partir de 60 dias após a poda (DAP). Antes de 60 DAP, na fase fenológica de brotação e crescimento vegetativo, a evapotranspiração foi elevada, devido à evaporação direta do solo. Após o início do crescimento vegetativo, o valor mínimo de $2,83 \mathrm{~mm} \mathrm{dia}{ }^{-1}$ ocorreu no período de 60 a 70 DAP (6 a 16 de agosto), compreendendo as fases de crescimento vegetativo e início de florescimento. $\mathrm{O}$ valor 
TABELA 1 - Fases fenológicas da goiabeira cv. Paluma em Petrolina - PE.

\begin{tabular}{cccc}
\hline fase & fase fenológica & período de 2001 & dias \\
\hline F1 & brotação e crescimento vegetativo & 7 de junho a 9 de agosto & 63 \\
F2 & crescimento vegetativo e florescimento & 10 a 23 de agosto & 14 \\
F2 & queda fisiológica dos frutos & 24 de agosto a 24 de setembro & 32 \\
F3 & crescimento dos frutos & 25 de setembro a 26 de novembro & 63 \\
F4 & maturação e colheita dos frutos & 27 de novembro a 24 de dezembro & total \\
& & 28 & 200 \\
\hline
\end{tabular}

máximo foi de $5,51 \mathrm{~mm} \mathrm{dia}^{-1}$ e ocorreu entre 150 e 160 DAP (4 a 11 de novembro), durante a fase fenológica de crescimento dos frutos, coincidindo com a época de grande demanda evapotranspiratória na região.

Os valores do Kc, obtidos com utilização de estações automática e convencional, apresentaram variações entre as fases de crescimento vegetativo (60 DAP) e o término da colheita dos frutos (200 DAP), podendo ser estimados por equações (Figura 2). Para a estação automática, o valor de Kc aumentou de 0,61 a 0,84, com média 0,73, enquanto, na convencional, de 0,75 a 0,93 , com média 0,84 . Os valores mínimos ocorreram na fase de crescimento vegetativo e os valores máximos na fase de crescimento dos frutos. Os menores valores de Kc da estação automática foram devidos aos maiores valores de ETo obtidos com a mesma estação.

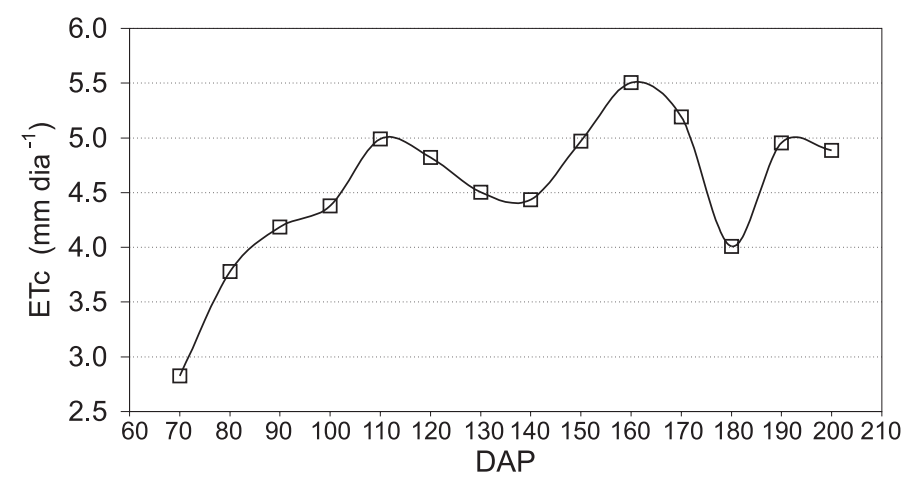

FIGURA 1 - Valores médios decendiais da evapotranspiração da cultura da goiabeira (ETc) em Petrolina - PE, em função dos dias após a poda (DAP).

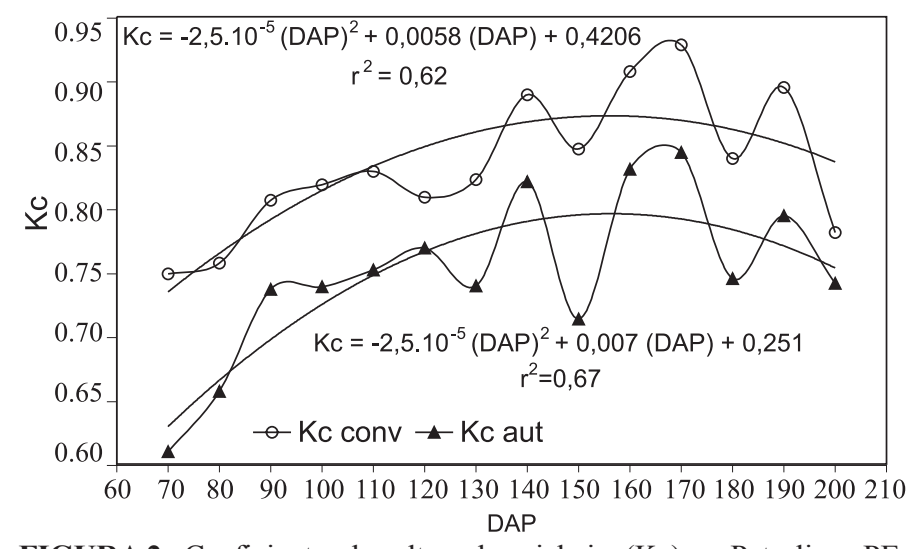

FIGURA 2 - Coeficientes de cultura da goiabeira (Kc) em Petrolina - PE, em função dos dias após a poda (DAP), obtidos com a utilização de estações agrometeorológicas automática (Kc aut) e convencional (Kc conv).

Devido às diferenças na precisão e da freqüência de amostragem dos sensores, para o cálculo dos valores médios, as estimativa de temperatura do ar, umidade relativa e radiação solar incidente por estações agrometeorológicas convencional e automática apresentam discrepâncias. Entretanto, os índices estatísticos mostram uma boa concordância entre os elementos observados pelas duas estações (Sentelhas et al., 1997).
No presente trabalho, os valores de temperatura e umidade do ar foram obtidos de maneiras diferentes, e ambos os elementos são considerados na determinação da pressão do vapor d'água ( $e_{a}$ e $e_{s}$; equações 11 e 12). A radiação solar incidente (Rs) também foi obtida de forma diferencial, e é considerada na estimativa do saldo de radiação (Rn; equações 8 a 10). Como o método de Penman-Monteith (equação 7) leva em consideração, entre outros, o déficit de pressão do vapor d'água ( $\mathrm{e}_{\mathrm{s}}$ - e e o saldo de radiação (Rn), os valores de ETo obtidos dessas estações apresentam discrepâncias; conseqüentemente, os valores de Kc são diferentes durante o ciclo da cultura (Figura 2).

A relação entre os valores de Kc obtidos com a estação automática e a estação convencional pode ser descrita por uma equação (Figura 3). Isto demonstra a possibilidade de correção nos valores desse parâmetro obtidos com estação agrometeorológica convencional, para uso com valores de ETo provenientes de estação automática. Isto pode ser explicado pela boa correlação de elementos climáticos medidos pelas estações convencionais e automáticas, como observado por Sentelhas et al. (1997)

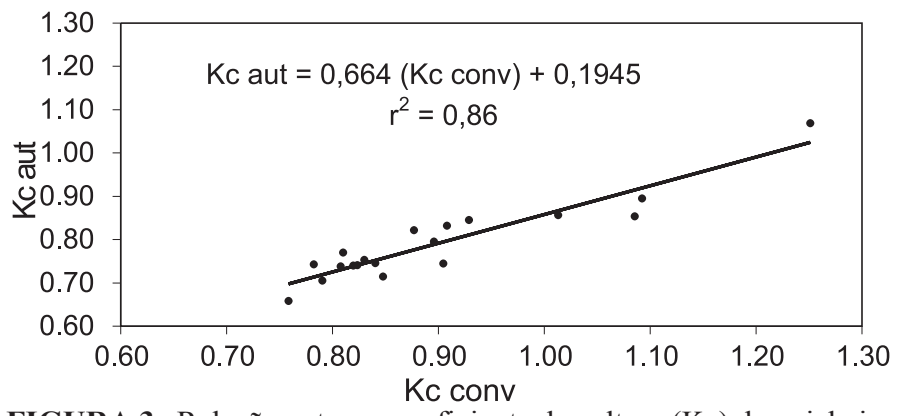

FIGURA 3 - Relação entre os coeficiente de cultura $(\mathrm{Kc})$ da goiabeira estimado pelas estações agrometeorológicas automática (Kc aut) e convencional (Kc conv).

Moura (2001) determinou o consumo de água e o Kc para a goiabeira cv Paluma irrigada em Petrolina-PE, aos 2 anos e 8 meses após o plantio, no espaçamento de 6 × $6 \mathrm{~m}$, e conduzida no sistema "poda sobre poda" ou poda contínua. Esse tipo de condução caracteriza-se pela poda de ramos em que os frutos já foram colhidos para o início de outro ciclo, concomitante com a existência de ramos com frutos na fase de maturação na mesma planta. Conseqüentemente, são encontrados frutos em vários estádios de crescimento. Assim, os valores de Kc obtidos com dados de estação agrometeorológica convencional apresentaram menor amplitude $(0,76$ a 0,81$)$ que os apresentados nesse trabalho, sendo os valores mínimo e máximo de ETc de 2,9 e $6,3 \mathrm{~mm} \mathrm{dia}^{-1}$, respectivamente.

\section{CONCLUSÕES}

Na estimativa da ETc da goiabeira, devem-se considerar os valores de Kc específicos para as estações agrometeorológicas convencional e automática, devido às diferenças nas estimativas de ETo. Entretanto, ao longo do ciclo da goiabeira, ocorre um comportamento similar desses coeficientes obtidos com dados das duas estações, com alta correlação entre ambos. Portanto, podem-se corrigir os valores de Kc obtidos pela estação convencional, quando se dispõe de dados de ETo 
provenientes da estação automática.

\section{REFERÊNCIASBIBLIOGRÁFICAS}

Allen, R. G., PEREIRA, L. S., RAES, D., SMith, M. Crop evapotranspiration: guidelines for computing crop water requirements. Rome:FAO, 1998.300p. (Irrigation and drainage, 56).

BASSOI, L.H.; TEIXEIRA, A.H.C.; SILVA, J.A.M.; SILVA, E.E.G;TARGINO, E.L.; MAIA, J.L.T.; FERREIRA, M.N.L. Parâmetros para o manejo de irrigação da goiabeira no Vale do São Francisco. In: CONGRESSO BRASILEIRODEENGENHARIAAGRÍCOLA, 31. 2002. Salvador. Anais... Salvador: SBEA, 2002. (CD-Rom).

CODEVASF. Cadastro Frutícola do Vale do São Francisco. Brasília: CODEVASF, 1999.(CD-Rom)

GONZAGANETO, L.; BEZERRA, J. E. F.; PEDROSA,A.A.C.; DANTAS, A.P.; SILVA, H.M. Comportamento produtivo da goiabeira sob irrigação no vale do Rio Moxotó. I. Variedades industriais: onze anos de produção. Revista Brasileira de Fruticultura, Cruz das Almas, v.13, n.3, p.103-114, 1991.

MOURA, S.B.B. Fenologia e consumo hídrico da goiabeira (Psidium guajava L.) irrigada, 2001. 124f. Dissertação (Mestrado em
Meteorologia), Universidade Federal da Paraíba, Centro de Ciências e Tecnologia, 2001.

PEREIRA, J.M.deA.; SOUZA, R.A. de. Mapeamento detalhado da área da Barra de Bebedouro. Recife: SUDENE, 1967.57p.

SENTELHAS, P.C.; MORAES, S.O.; PIEDADE, S.M.S.; PEREIRA, A.R.; ANGELOCCI, L.R.; MARIN, F.R. Análise comparativa de dados meteorológicos obtidos por estações convencional e automática. Revista Brasileira deAgrometeorologia, Santa Maria, v.5, n.2, p.215221, 1997.

TEIXEIRA, A. H. de C.. Relação entre a radiação solar global e insolação no município de Petrolina-PE. In: CONGRESSO BRASILEIRO DE AGROMETEOROLOGIA, 11., REUNIÃO LATINO-AMERICANA DE AGROMETEOROLOGIA, 2., 1999. Florianópolis. Anais... Florianóplis: Sociedade Brasileira deAgrometeorologia, 1999.p.798802.

WEBB, E. K. Aerial microclimate. Meteor ological Monographs, Boston, v. 6, n. 28, p. 27-58, 1965.

VAN GENUCHTEN, M.T. A closed-form equation for predicting the hydraulic conductivity of unsaturated soils. Soil Science Society of American Journal, Madison, v. 44, n.1, p. 898-982, 1980. 